\title{
Evaluation for stable resistance to Stenocarpella maydis in tropical maize (Zea mays $\mathbf{L}$.
}

\author{
Kelvin Simpasa ${ }^{1}$, Herbert Masole $^{2}$, Langa Tembo $^{1 *}$ \\ ${ }^{1}$ University of Zambia, Department of Plant Science, Box 32379 Lusaka, Zambia \\ ${ }^{2}$ Seed Co Zambia International Limited Box 35310, Lusaka Zambia
}

\begin{abstract}
Maize ear rots caused by Stenocarpella maydis cause reduction in yield and quality of the maize due to the mycotoxins produced by the pathogen. Breeding for resistance is the most feasible option in managing ear rots. However, to obtain stable resistance to $S$. maydis has been a challenge partly due to effect of the environment and availability of different isolates. The objective of this research was therefore, to determine the effect of multiple isolate inoculations in breeding for resistance to $S$. maydis and to identify genotypes with stable resistance. Seven inbred lines were crosses in a $7 x$ 7 full diallel without reciprocals. The resultant crosses (21) and their parents (7) were planted and evaluated at two sites, Lusaka and Mpongwe, during the 2015/16 cropping season. The experiment was laid out as a randomized complete block design with 3 replications. Treatments were: (1) single inoculation with isolate A, (2) single inoculation with isolate $B$ and (3) a multiple inoculation of two isolates $A B$ and (4) control with no inoculation at all. The mean genotypic scores were found to be 5.52, 4.96, 5.50 and 1 for treatment 1, 2, 3 and 4 respectively. The t-test analysis revealed that treatment 1 had a higher mean disease severity score (5.52) as compared to treatment 2 (4.96) $(P<0.01)$. Equally mean for treatment 2 (4.96) and 3 (5.50) were significantly different $(P<0.01)$. However, there were no significant differences between mean disease severity score for treatment 1 and 3. This indicated that multiple isolate inoculations could give rise to inappropriate genetic information due to the possibility of antagonistic effect between isolates. The genotypes (P2 $x$ P4) and (P3 $x$ P6) crosses were found to have stable resistance to $S$. maydis. These exhibited consistent significant negative SCA effects $(P<0.05)$ in both locations.
\end{abstract}

Keywords-Maize, ear rot, Stenocarpella maydis, resistance, mycotoxin, Specific combining ability (SCA).

\section{INTRODUCTION}

Maize (Zea mays L) is the world's most grown cereal and it is predicted that by 2020 it will surpass both rice and wheat to become the number one cereal in the world (M'mboyi, et al., 2010). The sub-Saharan populace depends on maize (Zea mays L) as the main staple carbohydrate source (Fischer et al., 2014). Approximately
(15.7\%) 22 million hectares, of the 140 million hectares grown globally, accounts for sub-Saharan Africa (Pingali, 2001). Farmers consider maize, not only to be a major source of energy but also their main source of income.

Maize production is carried out in diverse climates because of its versatility and it is the most productive species of food plants (Dowswell et al., 1996). In terms of soil, maize can be grown in wide range of soils, ranging from deep fertile soils along river bottoms and lake basins to welldrained and easily worked upland soils (M'mboyi et al., 2010).

Maize production is hampered by a number of biotic and abiotic stress factors. The biotic constraints in maize production include insects, weeds and pathogenic infection (M'mboyi et al., 2010). Among the diseases, ear rot caused by an important fungal pathogen, Stenocarpella maydis causes yield losses of 10-50 \% (Vigier et al., 2001). In preand post-harvest maize, the occurrence of mycotoxins is of great concern as they tend to cause health disorders in both livestock and humans who consume contaminated grain (Munkvold and Desjardins, 1997; Miller, 2001).

To control ear rots, a combination of crop sanitation, good agronomic practices and timely harvesting have been used, but with limited success (Munkvold, 2003). To curb this vice, deployment of resistant genotypes through breeding is the most cost effective way especially for the resource poor farmers in Zambia. However, resistance to S. maydis is greatly affected by underlying issues of gene interactions and the type of germplasm under study (Mukanga et al., 2011; Tembo et al., 2013). Identification of genotypes with stable resistance across locations can be utilized as the source of resistance in genotypic combinations (Tembo et al., 2013). A higher number of resistant parental genotypes to $S$. maydis in mating combinations are likely to produce a larger proportion of stable resistant off-springs. However, in maize, underlying issues of epistasis and gene interaction may interfere with expected outcome (ElBadawy, 2012) and there is therefore need for individual off-spring evaluation. In addition, it should be realized that effectiveness of breeding for stable resistance may be influenced by the type of isolates and its interaction with the environment (Rossouw et al., 2009). Previous studies have established multiple inoculations of different ear rot pathogens, as not an appropriate breeding strategy due to 
antagonistic effects associated with these pathogens (Tembo et al., 2013). Little is known about the effect of multiple isolate inoculations of $S$. maydis in breeding for resistance. Therefore, there is need to investigate that effect. Further breeding for stable resistance will therefore depend on the reaction effect of isolates when multiple inoculated. A previous study indicated that multiple pathogen inoculation should be employed for stable resistance if there are synergetic effects among pathogens (Chilipa et al., 2016) while this cannot clutch for pathogen combination with antagonistic effect (Tembo et al., 2013). The specific objective of this study therefore was i) to determine the appropriateness of multiple isolate inoculation on maize ear in breeding for stable resistance to Stenocarpella maydis and ii) to identify genotypes with stable resistance.

\section{MATERIALS AND METHODS}

\section{Germplasm used in the study}

Seven white-kernel parental inbred lines with varying reactions to $S$. maydis (Table 1) were crossed in a $7 \times 7$ full diallel (without reciprocals) during the 2015/16 off season. A total of 21 progenies ( $F_{1}$ single cross hybrids) together with their parents (a total of 28 genotypes) were evaluated in this study. The inbred lines were crossed in the 2015/16 off season.

\section{Study sites management and experiment design}

The evaluation trials were planted in December 2015/16 cropping season at Lusaka ( $15^{0} 24^{\prime} \mathrm{S} ; 28^{0} 04^{\prime} \mathrm{E}$, altitude $1216 \mathrm{~m}$ ) and Mpongwe (13 32'S; $28^{\circ}$ 03E, altitude 1206 $\mathrm{m})$. Rainfall received during the $2015 / 16$ cropping season was approximately $811 \mathrm{~mm}$ and $897 \mathrm{~mm}$ at the trial sites in Lusaka and Mpongwe respectively. Standard agronomical practices such as weeding and fertilizer application were followed. Fertilizer was applied at each site as compound D (N $35 \%$; P $70 \%$; K $35 \%$ ) 350 kg/ha and $300 \mathrm{~kg} / \mathrm{ha}$ of top dressing, Urea $(46 \% \mathrm{~N})$. The trial layout was a randomized complete block design (RCBD), with three replications in each location. The plants were established in two- row plots, $5 \mathrm{~m}$ long and $0.75 \mathrm{~m}$ apart and $0.25 \mathrm{~m}$ between plants. Trials were hand planted with two kernels per hill and later thinned at two weeks to one plant after emergence to a uniform stand of 20 plants per $5 \mathrm{~m}$. The cobs were inoculated with single and multiple isolates of S. maydis approximately 3-4 weeks after midsilking stage (Clements et al., 2003). Details of how the pathogen was cultured and toothpick-inoculated are explained in the following sections.

\section{Pathogen isolation and culture}

Isolates used in the study were obtained from Region II, Lusaka (15' 24'S; 28 $04^{\circ}$ 'E) and Region III, Mpongwe $\left(13^{\circ} 32^{\prime} \mathrm{S} ; 2^{\circ} \mathrm{03E}\right)$ and were confirmed to be distinct in their base morphology colour and spore count per $\mathrm{mm}^{2}$ as per procedure by Dorrance et al., (1999) and Rossouw et al., (2009). Isolate from region II and III were denoted as Isolate $\mathrm{A}$ and $\mathrm{B}$ respectively

Potato dextrose agar (PDA) media was prepared by weighing $3.9 \%$ of PDA powder into glass bottles filled with $500 \mathrm{~mL}$ of distilled water in order to culture isolate $\mathrm{A}$ and $\mathrm{B}$. The mixture was boiled while stirring until the powder dissolved completely. The glass bottles with the solution were then transferred to an autoclave for sterilization. The bottles and contents were autoclaved for 15 minutes at $121^{\circ} \mathrm{C}$ at a pressure of $15 \mathrm{MPa}$. 50 millimeters of the PDA solution was later poured into each of the 50 jars under the film board and left to cool overnight. $30(5 \mathrm{~cm} \times 5 \mathrm{~cm}$ base and $8 \mathrm{~cm}$ height $)$ jars were plastic and $20(9.5 \mathrm{~cm}$ diameter, $10 \mathrm{~cm}$ height $)$ were glass. 10 petri dishes $8.5 \mathrm{~cm}$ in diameter and $1.3 \mathrm{~cm}$ height were also filled with PDA solution and left to cool overnight. The petri dishes were used for initial culturing of the pathogen.

\section{Toothpick-inoculum preparation}

Toothpick-inoculum preparation was done using the modified procedure by Chambers (1988). A composite sample of $S$. maydis colonized kernels from each region denoted isolate sample A (Region II, Lusaka) and B (Region III, Mpongwe) were each separately sterilized in domestic bleach of the JIK brand that contains $3.5 \%$ sodium hypochlorite $(\mathrm{NaClO})$ (Reckitt Benickiser South Africa (Pvty) Limited) solution for three minutes and then rinsed thrice in distilled water. The kernels were blotted on sterilized filter paper to dry and then 3-5 kernels were plated on petri dishes with $3.9 \%$ potato dextrose agar and incubated at $27-30{ }^{\circ} \mathrm{C}$. After 4-5 days the fungal growths from the separately inoculated isolate (A and $\mathrm{B}$ ) plates were sub-cultured and ready to be transferred to toothpicks after 5-7 days.

The toothpicks were initially sterilized by boiling in water for 20 minutes and later air dried to room temperature. The toothpicks were then transferred to glass and plastic bottles which were initially autoclaved for 15 minutes and left to cool to room temperature. The bottles were filled with freshly prepared potato dextrose agar (PDA) and left to cool overnight to room temperature. The toothpicks were transferred to the bottles by placing them in an upright position in the bottles under the fume board. The plastic bottles contained approximately 100 toothpicks while the glass jars had between 150-200 toothpicks. Fungal culture plugs from pure cultures of each isolate of $S$. maydis were placed in specific bottles containing sterile toothpicks for ten days to allow the pathogen to fully colonize the toothpicks. Fully colonized toothpicks were then air dried before inoculating the genotypes.

\section{Inoculation of test ears}

Inoculation was done by piercing through the base of the test ear at 3-4 weeks after mid silking stage. Four treatments were used. Thus treatment 1 , involved single 
inoculation using a toothpick colonized separately with isolate $\mathrm{A}$; treatment 2 was done using single inoculation with toothpick colonized with isolate $\mathrm{B}$ and treatment 3 , multiple inoculation $(\mathrm{AB})$ using two toothpicks colonized by two different isolates $\mathrm{A}$ and $\mathrm{B}$ as done by Tembo et al, 2013. Multiple inoculation was achieved by inserting, these two toothpicks $5 \mathrm{~mm}$ apart into the base of the ear. Treatment 4, control was left without any inoculation at all in the second row. For each treatment five (5) plants were considered for inoculation and these were separated by three un-inoculated plants which acted as borders. Artificial inoculation encourages symptom development and disease progression and thus five plants were considered enough for assessment of the disease.

Single inoculations were performed in the first row with each isolate inoculation separated by three non-inoculated plants. Multiple inoculations were performed in the second row of the plot with the remaining plants treated as control and border plants.

\section{Data collection and analysis}

The plants were harvested at maturity and data were collected. Disease severity score was determined visually from all the five (5) inoculated plants per treatment in $5 \mathrm{~m}$ long first two row plots. Each inoculated treatment per genotype was harvested separately and the plot number noted. Percentage ear rot (ER) was estimated visually using percentage of ear colonized by the pathogen from the point of infection and the mean severity ratings computed. The rating was done using modified procedure by Tembo et al., 2013 with an $S$. maydis severity rating score as follows: $1=0-25 \% ; 2=26-50 \% ; 3=51-74 \% ; 4=$ $75-84 \% ; 5=85-94 \% ; 6=95-99 \%$ and $7=100 \%$ (completely rotten).

A paired two tailed t-test, was performed to compare the mean differences for $S$. maydis diseases severity scores among the three treatments across locations (Treatments 1 [Inoculation with Isolate A], 2[Inoculation with Isolate B] and 3 [Multiple inoculation with Isolate A \& B]). This was performed in Microsoft excel 2010.

Diallel analysis was performed using Griffing (1956) method 2, model I, fixed model in GenStat using Restricted Maximum Likelihood (REML) and regression approaches. The relative importance of GCA (general combining ability) and SCA (specific combining ability) effects were estimated.

\section{RESULTS}

\section{Effect of multiple isolate inoculations}

The mean disease severity scores across genotypes for treatment 1 (inoculation with isolate $\mathrm{A}$ ), treatment 2 (inoculation with isolate B) and treatment 3 (inoculation with isolate A \& B) were 5.52, 4.96 and 5.50 respectively. A student's paired t-test (Table 2) performed on comparison of treatment 1 mean disease severity score comparison (MDSC) and treatment 2, indicated highly significant $(\mathrm{P}<0.001)$ mean differences. Significant mean disease severity score differences were equally found between treatment 2 MDSC and treatment 3 on disease severity.

\section{Stenocarpella maydis ear rots genotypic disease severity effect}

Significant differences were obtained among genotypes with regards to $S$. maydis disease severity scores across inoculation treatments in each location (Table 3) (P < 0.01). Similarly, across location data shows interaction between location $\mathrm{x}$ genotype and location $\mathrm{x}$ isolate were highly significant $(\mathrm{P}<0.01$ and 0.001 respectively). Further analysis per location revealed significant $(\mathrm{P}<$ 0.001) specific combining ability (SCA) effects across treatments in both locations.

The genotypic mean disease severity effects of each isolate vis-à-vis, treatment 1 , treatment 2 , treatment 3 and treatment 4 [as the control on the test genotypes] was found to be 5.52, 4.96, 5.50 and 1 respectively (Table 4). The individual hybrids crosses mean severity scores and there SCA effects are tabulated below (Table 5).

The hybrids (P2 x P4) and (P3 x P6) crosses were found to have stable resistance to $S$. maydis across locations. (P2 x P4) exhibited genotypic means of 3.79 and 2.92 for Lusaka and Mpongwe respectively. (P3 x P6) showed a genotypic mean of 3.65 for Lusaka and 2.88 for Mpongwe. The significant SCA effects for (P2 x P4) were -0.39 and -0.76 for Lusaka and Mpongwe respectively whereas (P3 x P6) exhibited significant SCA effects of 0.54 (Lusaka) and -0.86 (Mpongwe).

\section{DISCUSSION}

Breeding for stable resistance against ear rots such as $S$. maydis has been a challenge, primarily due to environmental factors. In addition, breeders have previously bred for resistance to $S$. maydis without particularly taking the aspects of isolates into consideration (Rossouw et al., 2002; Tembo et al., 2013). It remains to be established if isolates have an effect in breeding for stable resistance. It was for this reason that the effect of isolates in breeding for resistance was investigated in this research study. In this study Isolate A (obtained from Lusaka) and Isolate B (obtained from Mpongwe) were used.

A paired t-test revealed that mean disease severity scoring for treatment 1 (inoculation with isolate A) was higher than that for treatment 2 (inoculation with isolate B) (Table 2). The fact that the mean disease severity for treatment 3 was higher $(\mathrm{P}<0.01)$ than treatment 2 , but not significantly different from treatment 1 , indicates that isolate A could have suppressed the virulence effect of isolate B when multiple inoculated. Previous studies on ear rot pathogens discouraged multiple inoculations of ear 
rot pathogens as a breeding strategy because of the antagonistic effects (Tembo et al., 2013). This paper, reports the possibility of antagonistic effects of multiple isolate inoculations which have not been fully exploited recognizing that different isolates exists for $S$. maydis. However, contradictory information has been reported in sweet potato and common beans upon multiple infections with sweet potato virus (SPV) and Collectorichum lindemuthianum respectively, whereby in this scenario synergistic interactions occurred (Gibson et al., 1998; Gasura and Mukasa, 2010; Chilipa et al., 2016).

It can therefore be deduced that multiple inoculation can either create antagonistic or synergistic effects. Multiple inoculations among different pathogens or isolates of the same pathogen with synergistic effects can be reliable and a beneficial screening approach for breeders. On the other hand, multiple inoculation approach of pathogens with antagonistic effects generates less informative genetic information (Tembo et al., 2013). Across isolate performance to determine stable resistance of genotypes was chosen. This is because isolates occur naturally, hence having genotypes with stable resistance across isolates will be ideal in tackling this challenge and to enhance resistance.

Some genotypes were found to possess significant specific combining ability (SCA) effects in both locations. Specific combining ability (SCA) effects can also assist in ascertaining which parental materials can be utilized in hybridization. In Lusaka six crosses; (P1 x P4), (P2 x P4), (P3 x P7), (P6 x P7), (P3 x P6) and (P4 x P5) had negative significant (Table 5) SCA effects. This implied that these crosses exhibited higher resistance to $S$. maydis in their specific combinations when compared to other crosses with either one of the parents in common. In Mpongwe (P2 x P4) and (P3 x P6) had negative significant $(\mathrm{P}<0.05)$ SCA effects. $(\mathrm{P} 2 \times \mathrm{P} 4)$ and $(\mathrm{P} 3 \times \mathrm{x}$ P6) crosses were found to have stable resistance to $S$. maydis across locations and as such can be used as parents in three way crosses or marketed as single cross hybrids after further evaluation. These exhibited significant SCA effects in both trial locations (Lusaka and Mpongwe).

\section{CONCLUSION}

In breeding for resistance to $S$. maydis, multiple isolate inoculation technique was found to be inappropriate due to the possibility of antagonistic effects of the isolates as it could lead to misleading genetic information. The use of individual isolates in breeding for resistance to $S$. maydis will be ideal in this case. (P2 x P4) and (P3 x P6) crosses were found to have stable resistance to $S$. maydis across trial locations (Lusaka and Mpongwe).

\section{REFERENCES}

[1] Chambers KR, 1988. Effect of time of inoculation on Diplodia stalk and ear rot of maize in South Africa. Plant Disease, 72: 529-531.

[2] Chilipa LN, Lungu DM, Tembo L, 2016. Multiple race inoculation as an option in breeding for resistance to $C$. lindemuthianium in common beans. Journal of Agriculture and Crops, 45-50.

[3] Clements MJ, Kleinshmidit CE, Pataky JK, White DG, 2003. Evaluation of inoculation techniques for Fusarium ear rot and fumonisims contamination of corn. Plant Disease, 87: 147-153.

[4] Dowswell, C., Paliwal, R., \& Cantrell, R. P. 1996. Maize in the Third World. Boulder, Colorado: Westview Press.

[5] El-Badawy MEM. 2012. Estimation of genetic parameters in three maize crosses for yield and its attributes. Asian J. Crop Sci, 4: 127-138

[6] Fischer RA, Byerlee D, Edmeades ED, 2014. Crop yields and global food security: will yield increase continue to feed the world? Canberra: Australian Centre for International Agricultural Research.

[7] Gasura E, Mukasa SB, 2010. Prevelance and Implications of swee tpotato recovery from sweet potato virus disease in Uganda. African Crop Science Journal 10: 195-205.

[8] Gibson RW, Mpembe J, Alicia T, Carey EE, Mwanga RO, Seal SE, 1998. Symptoms, etiology and serological analysis of sweet potato virus diseases in Uganda. Plant Pathology 47: 95-102.

[9] Griffing B, 1956. Concept of general and specific combining ability in relation to diallel crossing systems. Australian Journal of Biological Sciences 9: 463-493.

[10] Miller JD, 2001. Factors that affect the occurrence of fumonisins. Environmental Health Perspectives, 109: 321-324.

[11] M'mboyi F, Mugo S, Mwimali M, Ambani L, 2010. Maize Production and Improvement in Sub-Saharan Africa. Nairobi: African Biotechnology Stakeholders Forum (ABSF).

[12] Mukanga, M., Derera, J., Tongoona, P., \& Laing, M. D. (2011). Farmers' perceptions and management of maize ear rots and their implications in breeding for resistance. Afr. J. Agric. Res, 6, 4544-4554.

[13] Munkvold GP, 2003. Cultural and Genetic Approaches to Managing Mycotoxins in Maize. DOI: 10.1146/annurev.phyto.41.052002.095510, 41, 99-116.

[14] Munkvold GP, Desjardins AE, 1997. Fumonisins in maize. Can we reduce their occurrence? Plant Dis, 81: 556-564. 
[15] Pingali PL, 2001. Meeting world maize needs: Technological opportunities and priorities for the public sector. Mexico, DF (Mexico): CIMMYT.

[16] Rossouw JD, Pretorius ZA, Silva HD, Lamkey KR. 2009. Breeding for Resistance to Stenocarpella Ear Rot in Maize. In Plant Breeding review. Hoboken: John Wiley and Sons.

[17] Rossouw JD, Van Rensburg JB, Van Deventer CS, 2002. Breeding for resistance to ear rot of maize, caused by Stenocarpella maydis (Berk) Sutton. South Africa Journal Plant Soil, 4: 188-194.

[18] Tembo L, Asea G, Gibson PT, Okori P, 2013. Resistance breeding startegy for Stenocarpella maydis and Fusarium graminearum cob rots in tropical maize. Plant Breeding, 132: 83-89.

[19] Vigier B, Reid LM, Dwyer DW, Stewart RC., Sinha JT, Butler G, 2001. Maize Resistance to Gibberella ear rot:Symptoms, deoxynivalenol and yield. Canada Journal of Plant Pathology, 23: 99-105.

TABLES

Table.1: Germplasm used in the experiment for S. maydis in Lusaka and Mpongwe

\begin{tabular}{llllll}
\hline Parent & Name & Type & Source & Grain text & Reaction to S. maydis \\
\hline P1 & XL 003 & Inbred line & Public & F & Resistant \\
P2 & XL 029 & Inbred line & Public & SF & Susceptible \\
P3 & XL 057 & Inbred line & Public & F & Resistant \\
P4 & XL 071 & Inbred line & Public & F & Susceptible \\
P5 & XL 083 & Inbred line & Public & SF & Moderate \\
P6 & XL 087 & Inbred line & Public & F & Resistant \\
P7 & XL 195 & Inbred line & Public & F & Resistant \\
\hline
\end{tabular}

Where P-parent line, Grain texture, F-flint, SF-semi flint

Table.2: Genotypic mean disease severity score comparisons (MDSC) among treatments

\begin{tabular}{lc}
\hline MDSC & Student t-test (P- Value) \\
\hline Treatment 1 $(5.52)^{\mathrm{x}}$ vs Treatment 2(4.96) & $<0.001$ \\
Treatment $1(5.52)^{\mathrm{x}}$ vs Treatment 3 $(5.50)^{\mathrm{z}}$ & 0.13 \\
Treatment $2(4.96)^{\mathrm{y}}$ vs Treatment 3 $(5.50)^{\mathrm{z}}$ & $<0.001$ \\
\hline
\end{tabular}

$\mathrm{x}, \mathrm{y}, \mathrm{z}$ mean disease severity score across genotypes for treatments 1, 2 and 3 respectively. Treatment 1, 2 and 3 represents treatments with: single inoculation with isolate A, Single inoculation with isolate B and multiple inoculations of Isolates A \& B respectively.

Table.3: Mean squares for Stenocarpella maydis cob rot disease severity scores across two experimental locations and in each individual location evaluated in 2015/16 season.

\begin{tabular}{|c|c|c|c|c|c|}
\hline \multirow[b]{2}{*}{ Source } & \multirow[b]{2}{*}{ df } & \multirow{2}{*}{$\begin{array}{l}\text { Across locations } \\
\text { Across locations }\end{array}$} & \multirow[b]{2}{*}{ df } & \multicolumn{2}{|c|}{ Individual sites } \\
\hline & & & & Lusaka & Mpongwe \\
\hline Location & 1 & $241.01 * *$ & & & \\
\hline Replication/location & 4 & & 2 & 4.01 & 11.56 \\
\hline Genotype & 27 & 4.69 & 27 & $2.26 * *$ & $5.40 * *$ \\
\hline GCA & & & 6 & 0.96 & 3.84 \\
\hline $\mathrm{SCA}$ & & & 21 & $2.64 * * *$ & $5.85 * *$ \\
\hline Isolate & 3 & $770.44 * * *$ & 3 & $557.54 * *$ & $247.82 * *$ \\
\hline Location x Genotype & 27 & $2.83^{* *}$ & 27 & & \\
\hline Location $\mathrm{x}$ isolate & 3 & $35.51 * * *$ & 3 & & \\
\hline Genotype $\mathrm{x}$ isolate & 81 & 1.80 & 81 & 0.62 & 2.52 \\
\hline Gen $\mathrm{x}$ Isolate $\mathrm{x}$ location & 81 & 1.35 & 81 & & \\
\hline Error & 444 & 1.06 & 222 & 0.5 & 2.02 \\
\hline
\end{tabular}

$* *$, *** significant at $\mathrm{P} \leq 0.01$, and $\mathrm{P} \leq 0.001$ respectively, $\mathrm{MS}$, mean square 
Table.4: Effect of treatments on the test genotypes across the locations during 2015/16 cropping season.

\begin{tabular}{l|l}
\hline Treatment & Mean \\
\hline Treatment 1 & 5.52 \\
Treatment 2 & 4.96 \\
Treatment 3 & 5.50 \\
Treatment 4 & 1.00 \\
LSD $(\alpha=0.05)$ & 0.23 \\
\hline
\end{tabular}

LSD, Fishers Protected Least Significant Difference test performed at $\mathrm{P} \leq 0.05$

Treatment 1, 2, 3 and 4 are: Single inoculation with isolate A, single inoculation with isolate B, multiple inoculations with isolate (A \& B) and control without any inoculation respectively.

Table.5: Mean disease severity scores across treatments to Stenocarpella maydis in Lusaka and Mpongwe during 2015/16

\begin{tabular}{|c|c|c|c|c|}
\hline \multirow[b]{2}{*}{ Cross } & \multicolumn{2}{|c|}{ Lusaka } & \multicolumn{2}{|c|}{ Mpongwe } \\
\hline & Mean & SCA Effect & Mean & SCA Effect \\
\hline P1XP2 & 3.86 & $-0.02^{\text {ns }}$ & 3.13 & $0.02^{\mathrm{ns}}$ \\
\hline P1XP3 & 4.11 & $0.04^{\mathrm{ns}}$ & 3.55 & $0.13^{\mathrm{ns}}$ \\
\hline P1XP4 & 4.06 & $-0.40 *$ & 3.53 & $-0.48^{n s}$ \\
\hline P1XP5 & 4.25 & $0.04^{\mathrm{ns}}$ & 3.42 & $-0.06^{\mathrm{ns}}$ \\
\hline P1XP6 & 4.44 & $0.17^{\mathrm{ns}}$ & 4.05 & $0.28^{\mathrm{ns}}$ \\
\hline P1XP7 & 4.17 & $0.17^{\mathrm{ns}}$ & 3.25 & $0.11^{\mathrm{ns}}$ \\
\hline P2XP3 & 3.92 & $0.12^{\mathrm{ns}}$ & 3.51 & $0.42^{\mathrm{ns}}$ \\
\hline P2XP4 & 3.79 & $-0.39 *$ & 2.92 & $-0.76^{*}$ \\
\hline P2XP5 & 3.81 & $-0.13^{\text {ns }}$ & 2.88 & $-0.27^{\mathrm{ns}}$ \\
\hline P2XP6 & 4.5 & $0.51 * *$ & 4.33 & $0.90 * *$ \\
\hline P2XP7 & 3.63 & $-0.09^{\mathrm{ns}}$ & 2.5 & $-0.30^{\mathrm{ns}}$ \\
\hline P3XP4 & 5.05 & $0.67 * * *$ & 4.68 & $0.70 *$ \\
\hline P3XP5 & 4.26 & $0.13^{\mathrm{ns}}$ & 3.34 & $-0.12^{\mathrm{ns}}$ \\
\hline P3XP6 & 3.65 & $-0.54 * *$ & 2.88 & $-0.86^{*}$ \\
\hline P3XP7 & 3.5 & $-0.42 *$ & 2.83 & $-0.28^{\mathrm{ns}}$ \\
\hline P4XP5 & 4.07 & $-0.45 * *$ & 3.98 & $-0.07^{\mathrm{ns}}$ \\
\hline P4XP6 & 4.61 & $0.04^{\mathrm{ns}}$ & 4.48 & $0.15^{\mathrm{ns}}$ \\
\hline P4XP7 & 4.83 & $0.53^{* *}$ & 4.17 & $0.47^{\mathrm{ns}}$ \\
\hline P5XP6 & 4.54 & $0.21^{\mathrm{ns}}$ & 3.83 & $0.03^{\mathrm{ns}}$ \\
\hline P5XP7 & 4.26 & $0.20^{\mathrm{ns}}$ & 3.67 & $0.49^{\mathrm{ns}}$ \\
\hline \multirow[t]{3}{*}{ P6XP7 } & 3.73 & $-0.38 *$ & 2.96 & $-0.50^{\mathrm{ns}}$ \\
\hline & $4.14^{\mathrm{x}}$ & $0.18^{y}$ & $3.52^{x}$ & $0.37^{\mathrm{y}}$ \\
\hline & $0.57^{z}$ & & $1.45^{\mathrm{z}}$ & \\
\hline
\end{tabular}

+ Crosses derived from parental inbreds P1 to P7 as described in Table 1. LSD, Fishers Protected Least Significant Difference test performed at $\mathrm{P} \leq 0.05$. . $\mathrm{x}$ - Grand locational mean. y- Standard error of the mean. z- Least Significant difference. 